$45 \mathrm{HD}$, and $37 \mathrm{PD}$ patients.

From the initial swab culture, vancomycin-sensitive enterococci (VSE) were isolated in 65\% of CRI, 54\% of HD, and $70 \%$ of PD patients. No CRI or HD patients had VRE isolated, and 2\% (1 of 50) of PD patients had VRE isolated. The remaining patients had no enterococci isolated. Review of antimicrobial exposures in the 6 months before the initial swab test showed $0 \%$ of CRI, $32 \%$ of $\mathrm{HD}$, and $36 \%$ of PD patients received vancomycin. Other antimicrobials were administered to $40 \%$ of CRI, $46 \%$ of $\mathrm{HD}$, and $78 \%$ of PD patients in the same time period. In the month immediately preceding the initial swab test, $0 \%$ of CRI, $12 \%$ of HD, and $22 \%$ of PD patients received vancomycin, and $18 \%$ of CRI, $20 \%$ of $\mathrm{HD}$, and $36 \%$ of $\mathrm{PD}$ patients received other antimicrobials. Results from repeated cultures showed that $57 \%$ of CRI, $40 \%$ of HD, and $38 \%$ of PD patients changed their culture status related to VSE, VRE, or no enterococci present. Cultures of 342 swabs from 140 patients yielded three VRE isolates in two patients.

The investigators concluded that, despite the frequent use of vancomycin and other antimicrobials, the incidence of VRE in their renal population was less than the overall reported incidence. Given this lack of VRE isolates, they recommend the continued judicious use of vancomycin in treating renal patients and continued enterococcal sensitivity surveillance.

From: Brady JP, Snyder JW, Hasbargen JA. Vancomycin-resistant Enterococcus in end-stage renal disease. Am J Kidney Dis 1998;32:415-418.

\section{Efficacy of a Chlorous Acid Preoperative Skin Antiseptic}

Among the ways used to attempt to reduce the incidence of surgical infections is the use of preoperative antiseptics. Iodophors and chlorhexidines are the primary presurgical germicides used currently. Aly and colleagues from the Department of Dermatology, University of California-San Francisco, have reported a newly developed preoperative skin antiseptic (Alcide Corporation, Redmond, WA) that involves the mixture of a $0.1 \%$ sodium chlorite formulation and an activating preparation of $0.5 \%$ mandelic acid to produce chlorous acid and other antimicrobial degradation products.

Under clinical conditions, the antimicrobial effectiveness of this blended preparation was compared with a chlorhexidine gluconate scrub solution. Using a Latinsquare randomization scheme, two contralateral abdominal sites and two contralateral inguinal sites of healthy male and female volunteers were treated with these antiseptics or the controls of vehicle or activator alone. Microorganisms were harvested and quantified by a standard cylinder-scrub technique.

Of 85 volunteers (170 possible sites per test region) screened to meet the Food and Drug Administration baseline criteria on population densities of normal flora, 61 abdominal and 107 inguinal sites provided suitable data. Against chlorhexidine, the chlorous acid presurgical skin preparation produced superior but statistically equivalent reductions $(P>.05)$ of each volunteer's normal flora at 10 minutes, 30 minutes, and 6 hours after treatment. As many as 4-log reductions were achieved, and activity persisted as long as 6 hours. Compared with chlorhexidine, the chlorous acid product had the practical advantages of a shorter scrub time, less foam, air drying (instead of wiping), and no perceivable residue.

The authors concluded that an activated chlorous acid product statistically matched the performance of chlorhexidine gluconate in reducing populations of resident flora on treated skin sites. With an easier and shorter application procedure and strong, long-term antimicrobial activity, the new halogenated antiseptic seems to be a useful and possible efficacious preoperative skin disinfectant.

From: Aly R, Bayles C, Bibel DJ, Maibach HI, Orsine CA. Clinical efficacy of a chlorous acid preoperative skin antiseptic. Am J Infect Control 1998;26(4):406-412.

\section{Varicella Outbreak in Pediatric Oncology Unit}

During a nationwide varicella outbreak in Greece, Kavaliotis and coinvestigators from the Department of Paediatrics, Hospital for Infectious Diseases, Thessaloniki, Greece, studied the epidemiology and the efficacy of antiviral treatment and immunoprophylaxis for varicella-zoster virus (VZV) infection. This infection is usually benign, but immunocompromised patients are at great risk for visceral dissemination and fatal outcome.

During a 9-month period, 52 patients were exposed to cases of active varicella. Twenty-seven of these children were re-exposed to active varicella $>1$ month after their initial exposure. In all cases, prophylaxis with intravenous immunoglobulin (IVIG), varicella zoster globulin (VZIG), or both was given. The spread of the disease was limited, and only 6 patients (all immunosuppressed) developed varicella $(7.6 \%)$. Three of 6 had been given IVIG and 3 VZIG + IVIG. All patients with varicella received acyclovir $30 \mathrm{mg} / \mathrm{kg} /$ day for 14 days. The disease was mild, and all patients were cured.

The results show that prophylaxis was not $100 \%$ effective but appeared to reduce the rate of spread. The differences in incidence among the regimens used were not significant. It was concluded that immunoprophylaxis and acyclovir administration appear to be satisfactory in managing immunocompromised children exposed to VZV. This may change with the wider use of the varicella vaccine.

From: Kavaliotis J, Loukou I, Trachana M, Gombakis $\mathrm{N}$, Tsagaropoulou-Stigga H, Koliouskas D. Outbreak of varicella in a pediatric oncology unit. Med Pediatr Oncol 1998;31:166-169.

Additional news items in this issue: Potential for Cross-Contamination With Needleless Injector, page 850; Tea for Staphylococcus? page 850; OSHA Requests Information on Needlesticks, page 871. 